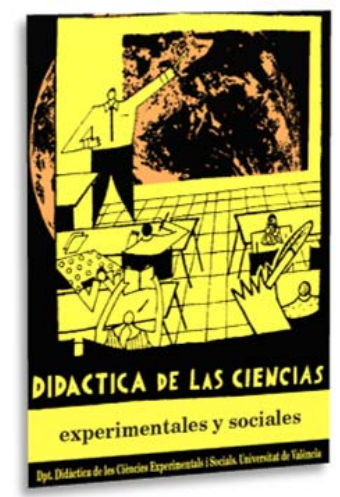

\title{
Feminismo y percepción del sexismo mediante TIC en educación superior. Un estudio de caso
}

\author{
Feminism and perception of sexism through \\ ICT in higher education. A case study
}

DOI: $10.7203 / D C E S .36 .12634$

\author{
Daniel David Martínez Romera \\ Universidad de Cádiz, danieldavid.martinez@uca.es \\ ORCID iD: http://orcid.org/0000-0003-4895-7955
}

\begin{abstract}
RESUMEN: Encontrar nuevas vías para trabajar temas sensibles en Didáctica de las Ciencias Sociales, como el que se propone, es tanto un reto como una necesidad docente, especialmente en contextos como el seleccionado (Educación para la Ciudadanía). Es por ello que se presenta un estudio diseñado para comprobar si las TIC pueden utilizarse de forma positiva en la exposición, debate y análisis de argumentos en ámbitos formales, así como para lograr un mayor grado de expresión individual de las posiciones propias. Aunque es necesario desarrollar más estudios en esta línea, los resultados de la experiencia han permitido plantear y discutir los contenidos del tema con un grado de participación y con unas posibilidades analíticas muy prometedoras. De forma adicional, ha sido posible incluir nuevas perspectivas como el análisis de subgrupos o la relación con la estructura temporal de los contenidos trabajados.
\end{abstract}

Palabras Clave: Feminismo, Sexismo, Ciencias Sociales, TIC, Didáctica.

ABSTRACT: Finding new ways to work on sensitive topics in Social Science Didactics, like the one proposed, is both a challenge and a teaching need, especially in contexts such as the one selected (Education for Citizenship). That is why a study was designed to check whether ICT can be used positively in the presentation, debate and analysis of arguments in formal areas, as well as to achieve a greater degree of individual expression of personal positions. Although it is necessary to develop more studies in this line, the results of the experience have allowed to raise and discuss the contents of the subject with a degree of participation and with very promising analytical possibilities. Additionally, new perspectives such as the analysis of subgroups or the relationship with the temporal structure of the worked contents have been included.

KEYwORDs: Feminism, Sexism, Social Sciences, ITC, Didactics.

Fecha de recepción: junio de 2018 Fecha de aceptación: diciembre de 2018

El presente trabajo se enmarca dentro del proyecto de innovación educativa interuniversitario "Aplicando la interdisciplinariedad en el aula: la mujer transitando espacios y rompiendo moldes (siglos XV-XX)" dirigido por Yolanda V. Olmedo, de la Universidad de Córdoba. 


\section{INTRODUCCIÓN}

El sexismo, entendiendo por éste la definición que ofrece la Real Academia de la Lengua, discriminación de personas por razón de sexo, es claramente una práctica contraria al humanismo educativo, propio del contexto universitario al menos en Ciencias de la Educación, lesiva de principios individuales y colectivos de convivencia; en este sentido, la Declaración Universal de los Derechos Humanos de 1948 es la referencia primera en el contexto de las democracias contemporáneas y nuestro punto de partida en la presente.

Desde entonces, han venido a sumarse a ésta un buen número de textos de similar pretensión universalista, entre los que destacamos: Declaración de los Derechos del Niño, 1959; Declaración sobre la Eliminación de la Discriminación contra la Mujer, 1967; Convención sobre la Eliminación de todas las formas de Discriminación contra la Mujer, 1981; Convención sobre los Derechos del Niño, 1989; Estatuto de Roma, por el que se establece la Corte Penal Internacional, 1998).

La jurisprudencia nacional, tomando como punto de partida la Constitución Española de 1978 y la ratificación de la citada convención de 1981, ha desarrollado desde entonces tres leyes orgánicas (LO) sobre la materia: de Medidas de Protección Integral contra la Violencia de Género (LO 1/2004, de 28 de diciembre); para la Igualdad Efectiva de Hombres y Mujeres (LO 3/2007, de 22 de marzo); de Salud Sexual y Reproductiva y de la Interrupción Voluntaria del Embarazo (LO 2/2010, de 3 de marzo). Así como ha ratificado el Convenio de Estambul (Boletín Oficial del Estado número 137, 6 de Junio de 2014) sobre prevención y lucha contra la violencia contra la mujer y la violencia doméstica.

Aun así, es fácil encontrar trabajos de diagnóstico o visibilización sobre comportamientos entroncados, en grado variable, con el sexismo (Diller, 2018; Gray, Blaise, y Knight, 2017; Jones \& Hughes, 2016; Maeso Fernández et al., 2015; Ronai, Zsembik, y Feagin, 2014), en los que se abordan sus causas, los contextos en que se producen y sus consecuencias individuales y colectivas. Esto pone de manifiesto, bajo nuestro criterio, que todavía es un tema con recorrido e interés social.

En este sentido, uno de los ámbitos que debe erigirse en referente de la superación de esta práctica es el educativo. En especial las Facultades de Educación, por tres motivos:

- La formación del profesorado no sólo debe ser lo más completa posible en términos epistemológicos y didácticos, sino también en valores y principios universales, ya que van a ser transmisores de cultura, en un sentido amplio.

- Por tradición histórica, al menos en España, estas facultades han sido espacios de transformación en las relaciones hombre-mujer. Además, se encuentran claramente feminizadas en su razón de sexos, por lo que son un excelente entorno para la medición de los avances sociales en esta cuestión.

- Al tratarse de población adulta joven, el alumnado universitario constituye un espacio de investigación ideal para el análisis comparado, la documentación y la realización de experiencias formativas; lo que puede ayudar en la detección de patrones de conducta, la construcción de modelos explicativos precisos de los mismos y la articulación de propuestas de acción para la superación de cualquier discriminación.

En el presente estudio de caso se diseña una experiencia para comprobar la utilidad de las tecnologías de la información y la comunicación (TIC) como instrumento de exposición, debate y recogida de datos en ámbito formal, sobre una cuestión tan sensible y difícil de medir como el sexismo. Sensible porque afecta al conjunto de valores personales, lo que lleva en última instancia al cuestionamiento de la propia ética. Difícil de medir porque al ser una cuestión social, está sujeta a los condicionantes del contexto, lo que incluye responder o expresar argumentos aceptables en el mismo, antes que coherentes con los propios valores. Así, para saber si una persona participa consciente o inconscientemente de una discriminación social, lo menos interesante desde el punto 
de vista de la investigación científica es preguntarle directamente por ello, si se pretende actuar con rigor (Cornejo y Salas, 2011).

Bajo nuestro punto de vista, el sexismo es el efecto y no la causa de determinados comportamientos. Por ello, también es necesario plantear y superar, definitivamente, un debate sobre algunos temas que hacen posible su génesis, desarrollo y persistencia: la imposición ideológica (Hammond, Milojev, Huang y Sibley, 2017), la resistencia al cambio (Jiménez Rodrigo, Román Onsalo y Traverso Cortés, 2011) y el derecho a no saber, la ignorancia cultivada como estrategia de éxito, aún hoy (Mingo y Moreno, 2015). Labor de análisis y reflexión que lleva décadas de estudio a sus espaldas, intentando articular propuestas eficaces de avance (Cámara Marín, 2014; Padilla Carmona, Sánchez García, Martín Berrido y Moreno Sánchez, 1999).

Este debate antecede al objetivo del presente estudio y supera en mucho las posibilidades de argumentación que podemos dedicar en esta introducción, dejaremos al menos establecidas nuestras directrices al respecto: todo acto educativo eficiente parte de las motivaciones e intereses del que aprende (Holt, 1983 y 1982); para que sea eficaz probablemente se requiera de un conflicto cognitivo en términos ausubelianos (Woolfolk, Winne, Perry y Shapka, 2010) o un atractor eficiente; así como de un espacio inviolable para que la integración y ampliación de conocimiento se lleve a cabo en términos de desarrollo personal autónomo y no de condicionamiento operante (Cepeda, Correa, Lozano y Urquizo, 2018). Esto, a nuestro juicio, coloca a la educación, la cultura y la sociedad en una situación en la que se deben tomar decisiones importantes (Fernández Enguita, 2016; Lerena, 1983).

\section{Metodología}

El estudio de caso se ha planteado en una asignatura bilingüe de Educación para la Ciudadanía y los Derechos Humanos del Grado de Educación Primaria de la Universidad de Málaga, durante el curso académico 2017/18. La población objeto de estudio fue el conjunto de la clase, 56 estudiantes, con presencia de 4 Erasmus provenientes de Corea del Sur, Finlandia, Holanda y Alemania. A pesar de su escaso número, poco más del 7\%, este último colectivo será analizado además con objeto de comparar la posible incidencia de los contextos culturales. Para simplificar la lectura, sólo se reproducirán literalmente y lengua inglesa las citas correspondientes a respuestas del alumnado utilizadas aquí para ilustrar el análisis.

El estudio comienza con un test previo para conocer el conocimiento que se tiene sobre el feminismo, en tanto que principal corriente heterogénea que asume el sexismo como una práctica social a cambiar. Se plantean 5 preguntas abiertas diseñadas para abarcar un espectro amplio de elementos clave de aquél, obtener la mayor cantidad de datos individuales que permitan comprender las posturas esgrimidas y evitar el riesgo de condicionamiento de respuesta (Nogueira, Nogueira, Navarro y Lizcano, 2015; Quivy y Campenhoudt, 2006). Las preguntas fueron (traducidas al español):

1. ¿Qué es el feminismo para ti?

2. ¿Cuáles son sus conceptos clave?

3. ¿Qué influencia/impacto tiene en la sociedad?

4. ¿Cuáles son las principales críticas que suele recibir el feminismo?

5. ¿Qué papel piensas que debería ocupar/tener en la Educación Primaria?

Con esto se pretende garantizar tres cuestiones críticas para el éxito del estudio: que la participación sea activa, a través de la expresión de los conocimientos y convicciones respecto al tema planteado; que la siguiente fase de trabajo se inicie con una actitud mental y de motivación adecuada; poder conocer un poco mejor el grado de conocimiento que posee la población estudiantil de la titulación al respecto. 
Tras esto, se plantea una fase breve de debate y recapitulación sobre las opiniones vertidas, que da paso al visionado, con software para la realización de anotaciones multimedia, de una pequeña charla TED, a cargo de Laura Bates, Everyday sexism. El contenido elegido lo ha sido por presentar cierto grado de polémica, tanto por el argumentario desarrollado por la autora, como por las críticas a favor y en contra recibidas dentro y fuera de movimientos en defensa de la mujer (Brown, 2015; Ali, 2013).

La pretensión es, en todo caso, garantizar que el alumnado, en un tiempo razonable, visione el contenido y emita, cuando estime oportuno, opiniones y réplicas a sus compañeros. Para facilitar la codificación de comentarios, se utiliza una lógica de etiquetas para clasificarlos en términos de a favor o en contra de lo que Bates explica (su proyecto web para la recogida de testimonios sobre sexismo), o la existencia de dudas al respecto. Se parte, en este sentido, de un desarrollo del apartado TIC que ha demostrado su utilidad sobre contenidos de la misma naturaleza (Martínez Romera, 2018), especialmente en cuanto a la jerarquización de etiquetas de contenidos: positivas, negativas, de duda, mixtas y omitidas.

Finalmente, el utillaje tecnológico se ha resuelto de la siguiente manera: para el análisis cuantitativo se ha utilizado IBM SPSS v.25 y para el cualitativos ATLAS.ti v.8.10. Ambas aplicaciones de reconocida trayectoria en el ámbito de la investigación educativa. Para el trabajo con anotaciones multimedia se ha recurrido a CoAnnotation, iniciativa pública del Grupo de Investigación en Globalización, Tecnología, Educación y Aprendizaje de la Universidad de Málaga (España).

\section{ANÁlisis}

El estudio cuantitativo del test previo ha implicado la depuración de más de ocho mil palabras, de las cuales significativas han resultado ser algo más de mil seteciencias. Se entiende por éstas las que son fruto de un triple filtrado:

- El descarte de palabras necesarias para una sintaxis correcta, de alta frecuencia, propias del idioma y no de la jerga de la materia objeto de estudio (preposiciones, determinantes, adverbios...).

- La simplificación de las formas de las palabras atendiendo a su raíz semántica o su significado como expresión, en su caso (ciudadanía, sociedad, sociedades, mundo, todos juntos).

- La eliminación de conceptos recurrentes que ya aparecen en la formulación de las preguntas; aunque son pertinentes, para la necesaria explicación de una postura es necesario recurrir a términos explicativos o calificativos complementarios o adicionales. En última instancia, es un mecanismo de control sobre argumentaciones tautológicas.

Todo ello realizado caso a caso, por participante y pregunta. Para poder tener una visión de conjunto del proceso, se presenta una tabla resumen, Tabla 1, con los principales descriptores cuantitativos, así como una criba conceptual por pregunta (asociadas a su frecuencia) y para el conjunto del test (palabras significativas más relevantes, en cursiva): 
TABLA 1. Resumen descriptivo de los resultados del test

\begin{tabular}{|c|c|c|c|c|c|c|}
\hline \multirow{2}{*}{ Palabras } & \multicolumn{5}{|c|}{ Pregunta } & \multirow{2}{*}{ Total } \\
\hline & 1 & 2 & 3 & 4 & 5 & \\
\hline Únicas & 1.182 & 858 & 2.068 & 1.760 & 2.295 & 8.163 \\
\hline Significativas & 213 & 187 & 494 & 368 & 476 & 1.738 \\
\hline Redundancia (\%) & 18,02 & 21,79 & 23,89 & 20,91 & 20,74 & 21,29 \\
\hline \multirow{3}{*}{$\begin{array}{l}\text { Conceptos } \\
\text { recurrentes por pregunta } \\
\text { (frecuencia) }\end{array}$} & mujer (84) & mujer (56) & mujer (50) & mujer (52) & educación (36) & mujer \\
\hline & hombre (47) & igualdad (54) & sociedad (48) & hombre (48) & igualdad (35) & hombre \\
\hline & igualdad (36) & hombre (26) & hombre (25) & sociedad (33) & mujer (35) & igualdad \\
\hline \multirow{3}{*}{$\begin{array}{l}\text { Conceptos modales en } \\
\text { el conjunto del test } \\
\text { (última columna) }\end{array}$} & movimiento (25) & género (16) & $\begin{array}{l}\text { feminismo } \\
(16)\end{array}$ & pensar (31) & roles (24) & sociedad \\
\hline & derechos (18) & respeto (6) & derechos (12) & $\begin{array}{l}\text { feminismo } \\
(32)\end{array}$ & pensar (20) & pensar \\
\hline & lucha (11) & sexismo (6) & pensar (12) & crítica (18) & alumnado (17) & movimiento \\
\hline
\end{tabular}

Fuente: elaboración propia

El porcentaje de redundancia es un cálculo básico del peso relativo de las palabras clave respecto a la respuesta en su conjunto, de modo que cuanto más elevado es el mismo, mayor frecuencia de aparición han tenido las segundas, en alguna de sus formas. Un valor elevado de este indicador refleja que la respuesta utiliza un número reducido de conceptos para argumentar la postura vertida, mientras que un valor bajo refleja, al menos en primera instancia, una mayor diversidad de términos en la articulación de la misma. Se considera una forma directa de medir la capacidad argumentativa y el dominio de un tema de discusión (Gyllstad, Vilkaité y Schmitt, 2015; Schmitt, 2014; Alavis y Akbarian, 2012).

Si bien en el ámbito de la Educación para la Ciudadanía no existen referencias conocidas sobre dichos umbrales, basándonos en el análisis individualizado y teniendo como referencia un corpus disciplinario acotado (Pilcher y Whelehan, 2016) del que han emergido los conceptos recurrentes de la tabla anterior, consideramos que los valores de redundancia en las preguntas (18 a 24\%) son medio-altos. Pues en términos generales reflejan un cierto conocimiento sobre la materia, pero sin llegar a hacer uso amplio de conceptos relacionados o complementarios.

En conjunto, la mayoría de conceptos clave de cada pregunta encuentra su reflejo en la obra de Pilcher y Whelehan (op. Cit.); de hecho, las palabras más relevantes del test (mujer, hombre, igualdad, sociedad, pensar y movimiento) tienen un reflejo directo (como igualdad), equivalente (sociedad>>ciudadanía), forma parte de ellos (hombre>>patriarcado; movimiento>>como una de las 'olas' del feminismo) o engloba (pensar>>punto de vista e ideología) el glosario propuesto por las autoras.

Sobre la primera pregunta, se ha manifestado una postura común bastante clara, por la cual el feminismo entiende a la mujer y el hombre como iguales. A este respecto no se han observado especiales discrepancias entre el colectivo Erasmus (primer ejemplo) y el resto de clase (segundo ejemplo). En todos los casos ilustrativos que se presentan, se subrayan los conceptos clave y se marcan en cursiva las reiteraciones o palabras asimilables a conceptos clave. Al ser citaciones exactas se evita introducir correcciones de lenguaje o modificación alguna:

- "It is a social movement of thought that strives for equality between men and women and to recognize the rights and duties that were recognised only in men for years."

- "Is the women's social movement in order to fight for their rights and freedoms and to get an iqual situation respect to men in the society." 
Las respuestas planteadas en términos de proyección teleológica también han estado presentes, pero se las considera respuestas que evitan objetivo real de la pregunta:

- "It is something that our society must keep going."

La segunda pregunta ha sido la más concisa de las cinco, en parte fruto de su formulación (que orienta la respuesta hacia el planteamiento de lista de conceptos) y en parte porque el resultado de esta estrategia ha descrito una situación general de escasa profundidad conceptual. Aquí, la situación entre estudiantes de Erasmus y el resto de clase vuelve a ser similar (primer y segundo ejemplo respectivamente), si bien la mayor densidad del segundo grupo permite encontrar en él respuestas más profundas (tercer ejemplo):

- "Equality of men and women, rights of women"

- "The key concepts are: equality, respect, security, rights, education.”

- "gender equality, coeducation, empowerment, gender empowerment, gender identity, machismo, patriarchy”

Llama poderosamente la atención que un concepto como patriarcado sólo fuera citado en 2 de los 56 casos y el de empoderamiento de género sólo fuese usado en el caso que se cita como ejemplo.

La tercera pregunta fue diseñada para conocer la valoración personal que le dan al feminismo como factor transformador de la sociedad. Aquí hubo cierta confusión, o repetición, en relación a la siguiente pregunta. Ya que algunas respuestas se orientaron a evaluar las críticas que aquel recibe cuando esta cuestión se aborda específicamente justo después. En todo caso, en términos generales, las respuestas se distribuyen en un rango que va desde el despertar conciencia de reivindicación, especialmente entre las mujeres, a concederle escasa influencia y concebirla como una estrategia a largo plazo, de avance lento. Además, son frecuentes las matizaciones geográficas, reconociéndose peores situaciones fuera del mundo occidental.

Sólo hay un caso, de Erasmus, que, tras matizar el contexto geográfico, entiende que hoy día no hay agravios comparativos entre hombres y mujeres, que éstos han sido superados (primer ejemplo), mientras que el resto citan su papel en la superación de las mismas (segundo ejemplo):

- "In the western society, woman's have the same rights as men"

- "Little by little every person on society have the same rights, and particulary women are more visible on many aspects of society in which they had no rights before.”

La cuarta pregunta persigue conocer el grado de conocimiento que tiene el alumnado del feminismo en el debate social. Aquí se produce una alta recurrencia de argumentos ligados al supremacismo femenino asociado a conceptos como hembrismo o feminazi; y, por otro lado, se señala que la lucha por la superación de agravios comparativos es percibida por el statu quo, como un ataque sin razón, por la que trata de desacreditarlo. Existe, finalmente, un tercer grupo, que considera que el vocablo de partida ya introduce un sesgo no integrador que dificulta la superación real de los agravios, algo especialmente llamativo dado que esta idea está más presente en mujeres que en hombres. Para cada uno de los casos citados podemos encontrar lo siguientes ejemplos:

- "Like all ideological movements, feminism is receiving criticism from all sectors of society. With this criticism, the increasingly popular term "feminazi" has emerged. Feminism is accused of not fighting for the rights of men, and that it is a movement for the supremacy of women."

- "People used to say that feminism does not mean equality for men and women. They think that people who are feminist they preffer women, is like machism. But it does not mean that, so they have to learn that this meaning is equality."

- "Feminism is not the solution, the term is not fair for men, faminism is not equality, it puts women above all men."

La quinta pregunta es la que mayor extensión textual ha tenido en conjunto; además es la más personal de todas, tanto porque se requiere abiertamente un juicio personal sobre el feminismo, como por el objeto con el que se relaciona, la educación primaria, de la que son futuros docentes. 
Aquí es importante tener en cuenta que ninguno de los estudiantes de Erasmus procede de una trayectoria formativa equivalente a profesorado de educación primaria, sino de materias tan diversas como economía, artes plásticas o periodismo. De modo que la comparativa no cumple parámetros elementales de pertinencia.

En todo caso, el grueso de los participantes manifiesta una postura común sobre la importancia de introducir elementos vigentes del discurso social en el aula, que sirva para la mejora de los valores comunes; sin embargo, también algunos casos matizan esto y señalan el riesgo del adoctrinamiento o el de delegar en la educación lo que también afecta al contexto familiar. Desde un punto de vista educativo, se lo relaciona también con la coeducación. Ejemplos ilustrativos de las dos matizaciones anteriores podrían ser:

- "I think teachers can treat this concept in a natural way and not see it as something controversial. It is important that students start knowing the value of the women since they are little."

- "I think that in school should be taught not feminism or sexism, not a difference between men and women, in school should be taught to respect both genders and the importance of both in society."

Por su parte, el análisis audiovisual que sucedió al test arroja lo siguientes datos (Tabla 2):

TABLA 2. Resumen descriptivo-cuantitativo de los comentarios a la charla de Laura Bates

\begin{tabular}{|c|c|c|c|c|c|c|c|c|c|c|c|c|c|}
\hline \multirow{2}{*}{ ID } & \multicolumn{6}{|c|}{ Etiquetas } & \multirow{2}{*}{ ID } & \multicolumn{6}{|c|}{ Etiquetas } \\
\hline & $\mathbf{P}$ & $\mathbf{N}$ & D & $\mathbf{M}$ & O & $\sum$ & & $\mathbf{P}$ & $\mathbf{N}$ & D & $\mathbf{M}$ & $\mathbf{O}$ & $\sum$ \\
\hline 1 & 1 & 0 & 0 & 6 & 0 & 7 & 27 & 6 & 0 & 0 & 0 & 0 & 6 \\
\hline 2 & 2 & 4 & 0 & 0 & 0 & 6 & 28 & 1 & 1 & 0 & 1 & 0 & 3 \\
\hline 3 & 0 & 3 & 0 & 0 & 1 & 4 & 29 & 0 & 5 & 0 & 0 & 2 & 7 \\
\hline 4 & 2 & 3 & 1 & 0 & 0 & 6 & 30 & 0 & 0 & 0 & 0 & 1 & 1 \\
\hline 5 & 2 & 2 & 1 & 0 & 0 & 5 & 31 & 1 & 0 & 0 & 0 & 0 & 1 \\
\hline 6 & 0 & 2 & 0 & 0 & 0 & 2 & 32 & 0 & 6 & 0 & 0 & 0 & 6 \\
\hline 7 & 0 & 1 & 0 & 0 & 0 & 1 & 33 & 2 & 9 & 0 & 0 & 0 & 11 \\
\hline 8 & 1 & 3 & 0 & 0 & 0 & 4 & 34 & 2 & 2 & 1 & 0 & 0 & 5 \\
\hline 9 & 2 & 5 & 0 & 0 & 0 & 7 & 35 & 0 & 0 & 0 & 2 & 0 & 2 \\
\hline 10 & 1 & 4 & 0 & 0 & 0 & 5 & 36 & 2 & 3 & 0 & 0 & 1 & 6 \\
\hline 11 & 2 & 3 & 0 & 0 & 1 & 6 & 37 & 6 & 0 & 1 & 0 & 0 & 7 \\
\hline 12 & 1 & 0 & 0 & 0 & 0 & 1 & 38 & 0 & 5 & 0 & 0 & 0 & 5 \\
\hline 13 & 0 & 0 & 0 & 3 & 0 & 3 & 39 & 1 & 0 & 0 & 0 & 0 & 1 \\
\hline 14 & 2 & 1 & 0 & 0 & 0 & 3 & 40 & 0 & 2 & 0 & 0 & 0 & 2 \\
\hline 15 & 1 & 0 & 2 & 0 & 0 & 3 & 41 & 4 & 0 & 0 & 0 & 0 & 4 \\
\hline 16 & 0 & 2 & 0 & 1 & 0 & 3 & 42 & 0 & 1 & 0 & 0 & 5 & 6 \\
\hline 17 & 0 & 3 & 0 & 0 & 0 & 3 & 43 & 0 & 1 & 0 & 0 & 2 & 3 \\
\hline 18 & 1 & 0 & 0 & 0 & 0 & 1 & 44 & 0 & 5 & 0 & 0 & 0 & 5 \\
\hline 19 & 0 & 0 & 0 & 0 & 6 & 6 & 45 & 2 & 2 & 0 & 0 & 0 & 4 \\
\hline 20 & 0 & 0 & 0 & 0 & 3 & 3 & 46 & 0 & 0 & 0 & 0 & 3 & 3 \\
\hline 21 & 2 & 0 & 1 & 0 & 0 & 3 & 47 & 0 & 0 & 0 & 0 & 7 & 7 \\
\hline 22 & 4 & 0 & 2 & 0 & 0 & 6 & 48 & 0 & 4 & 0 & 0 & 1 & 5 \\
\hline 23 & 2 & 0 & 0 & 0 & 0 & 2 & 49 & 0 & 3 & 0 & 0 & 1 & 4 \\
\hline 24 & 1 & 3 & 0 & 0 & 0 & 4 & 50 & 0 & 2 & 0 & 0 & 2 & 4 \\
\hline 25 & 5 & 0 & 0 & 0 & 0 & 5 & 51 & 0 & 0 & 0 & 0 & 3 & 3 \\
\hline 26 & 2 & 0 & 0 & 0 & 0 & 2 & $\sum$ & 61 & 90 & 9 & 13 & 39 & 212 \\
\hline
\end{tabular}

Fuente: elaboración propia. Nota: 91\% de cobertura, debido a 5 bajas durante la asignatura.

El trabajo medio es de 4.1 anotaciones por persona, de las cuales 1.18 serían positivos, 1.74 negativos, 0.18 expresarían duda, 0.25 estarían clasificados en más de una categoría y 0.75 no 
habrían hecho uso de las etiquetas. Éste último dato es especialmente relevante, ya que se trata de un valor significativo: el 18.3\% de todas las anotaciones fueron hechas sin clasificación, lo que en última instancia puede considerarse un defecto de forma, pero también un indicador del grado de comprensión de la tarea asignada. La distribución temporal de las categorías, a lo largo de los 20’ que duró la charla realizada por la autora para TEDxOxford fue la siguiente (Gráfico 1):

GRÁFICO 1. Distribución temporal de la frecuencia de tipos de anotación.

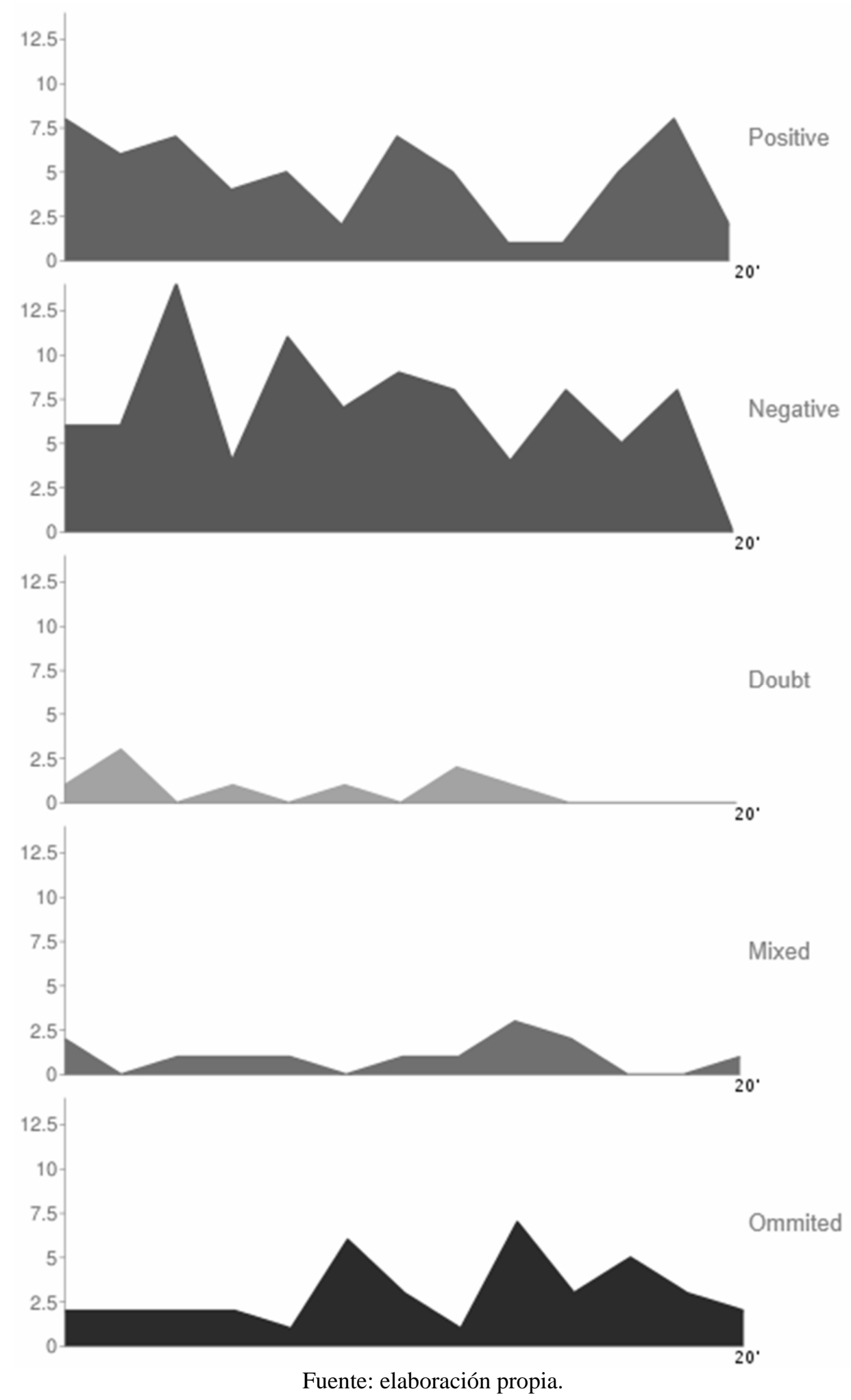


La distribución de comentarios positivos está presente durante toda la charla, encontrándose su mínimo al inicio del último tercio y su máximo en mitad de éste, unos minutos antes de que termine la charla. La distribución de comentarios negativos está igualmente presente en todo momento, si bien de una forma más enfatizada y con un máximo absoluto al final del primer tercio; tiene por solución de continuidad uno de los dos máximos absolutos presentes (final del primer tercio y final del segundo tercio).

Las anotaciones que expresan duda o presentan clasificación mixta son puntuales, de modo que durante partes importantes de la charla no están presentes, especialmente en el primer caso. En éste, además, el máximo absoluto se encuentra a los pocos minutos de iniciarse la charla, mientras que en aquel coincide prácticamente con el segundo mínimo absoluto de comentarios negativos. El quinto caso, comentarios que omiten etiqueta, es de facto el tercero en representatividad, lo que obliga a un estudio pormenorizado de la misma pues se trata de una verdadera caja negra.

En cuanto al contenido de las opiniones vertidas, de forma resumida e ilustrada por ejemplostipo, podemos establecer la siguiente estructura (respetando la lógica de tercios temporales utilizada):

\section{- Positivos}

o Inicio. Valoran positivamente la iniciativa presentada, considerándola pertinente y necesaria: "This project is a good example of how today's difficult issues can be tackled anonymously and reliably in a variety of ways in the Internet. People gather together, share their concerns and combine their strength.”

o Desarrollo. Reconocen los casos expuestos como realidades cercanas, motivo por el que en ocasiones se introducen cuestionamientos sobre la falta de visibilidad de los mismos y la pervivencia de prácticas incompatibles con los valores cívicos actuales: “At this point of the talk, I begin to wonder why nothing that has appeared on the screen or the stories she has told surprises me too much. Unfortunately, although I have not lived it by myself, the week of the rookies and the pranks are something that still exists in many universities and that is seen as something normal, a way to have fun totally legal. I am totally agree with the approach that the speaker has given to the issue: these type of rituals go beyond a simple 'joke'.”

o Conclusión. Se valora positivamente la pertinencia de la propuesta y se subraya como un elemento significante la crudeza de las situaciones descritas, especialmente por su cercanía temporal, en muchos también casos emocional. Con frecuencia se señala como clave la cotidianidad de los hechos como factor explicativo de su trivialización: "I totally agree with her, we need to talk and stop all of these belittiling against women. We don't deserve this treat because we are women, we need to be respected in any kind of situation.”

- Negativos

o Observación general. Todas las anotaciones en esta categoría podrían denominarse falsos negativos, en la medida que suelen ser concomitantes con la opinión vertida por la conferenciante, salvo por el hecho de que expresan con mayor viveza indignación o denuncia del carácter vigente de los hechos. Será, en todo caso un punto a tratar en la discusión de resultados.

o Inicio. La mayor parte de las anotaciones plantean indignación o sorpresa negativa sobre el papel de la educación, señalándose en varios casos la educación superior como fuente de reproducción del discurso que legitima el sexismo: "It seems to me degrading that in areas as universities, women are treated as sexual objects."

o Desarrollo. En esta fase de la charla el grueso de las anotaciones redundan en una idea muy clara, la indignación y la denuncia o reclamación social de 
cambio: "Why is the gorvernment letting the university to put those slogans? Why don't they do anything to solve this? A lot of girls are suffering those practices and they aren't coffesing because they are scared."

o Conclusión. Surge en esta parte una cierta tendencia a la desazón, aunque se reconoce el valor de mérito de la propuesta y se realiza una valoración negativa del uso actual de las tecnologías orientadas a la sociabilización, ilustradas en la charla: "These slides are very shcoked. I cannot believe that things like that are on facebook as a part of rapes normalization. It is something that scared me if we think that there are people who can enjoy with this type of crime. In my opinion is very hard that thing like that can be on facebook without a report or a immediate lock of this type of accounts."

- Duda

o Las anotaciones de esta categoría reconocen el argumento al que se asocian, pero matizan la importancia de trabajar en todos los frentes para su superación: “The concept of 'Culture of violation' has shocked me. Why do we have to go to that extreme in which we have to create a concept to refer to all the sexual and non-respetable behaviours of our society? Whe should struggle to change it. It is in our hands, but changing a part of the people's mind is not easy. Both, men and women have to unify the strength to fight against this situation."

- Mixtos

o Pese al escaso número de anotaciones, es una de las categorías más heterogéneas en cuanto a su contenido. El elemento más recursivo, de forma directa o indirecta, es el de sorpresa respecto a la existencia de estos comportamientos también hacia los hombres como víctimas (debemos recordar que la experiencia se realiza en un aula muy feminizada): "From my point of view, this video gives us the opportunity to reflect on sexism. Nowadays, activities like "The Week of the nocive students" are carried out to normalise behaviours which undervalue women and consider them as sexual objects. What's more, girls who are victims of sexual harassment and assault, feel embarrassed and responsible when they are living this situation. As a result, most of them do not report it. It is important to say victims in the majority of cases are women, although sometimes there are sexism conducts against men."

- No etiquetados

o Valoración global. Amalgama de todas las categorías anteriores, en su mayoría asignables a una de ellas de forma clara, sí presenta algunas singularidades.

o Singularidad 1. Sólo en esta categoría la gente ha considerado pertinente introducir elementos externos al debate: "What do you think about the new campaign against the alcohol for teenagers?? It is outrageous. https://www.elespanol.com/social/20171117/262724468_0.html"

o Singularidad 2. Sólo en esta categoría se introducen en el debate hechos sucedidos en la sociedad española para enfatizar la situación: "The video starts with Laura saying that it hits the number of young girls who wrote her commenting on what they had happened. Unfortunately, It doesn't impact me because it is what I live in my day to day and every time we are more women and girls. It's very alarming that only $10 \%$ of women who have suffered sexual harassment dare denounce it. 50\% don't do it out of embarrassment. Unfortunately, I can understand it perfectly because on the one hand it is too shameful and anguished to tell It for fear that they believe us or not. 43\% don't denounce It because they believe that they will be blamed. Today's society is getting us to feel this way because they ALWAYS going to blame us in one way 
or another asking us uncomfortable questions such as: Did you drink?, Did you go alone?, how did you dress? Instead of blame the person who committed this crime, they blame us. I have happened several unpleasant situations related whith this video and the answer that I always receive is: "it's normal, if you were dressed like this" or "It is normal, that happened because you go alone at night."" The most unpleasant thing is that I listen It from the masculine and feminine sector, including my closest environment. Without going further, we just have to see how it is treating the rape that a girl suffered in the "San Fermines" party last year, which She is being judged instead of them."

\section{DisCUSIÓN}

El conocimiento previo que tiene sobre el feminismo el alumnado del estudio pone de manifiesto un grado claramente superficial del mismo, lo que entronca con el desfase contemporáneo entre los procesos sociales y los posicionamientos académicos (Quinn, Davies y Lubelska, 2017; Martínez-Alemán, Pusser y Bensimon, 2015).

En las respuestas ofrecidas para cada pregunta del test, la diversidad de conceptos ha demostrado ser escasa y relativamente redundante, por lo que tiene sentido plantear un trabajo inicial previo sobre esta cuestión como condición necesaria para poder debatir sobre el sexismo, o en términos más generales, la situación de represión o agravio entre sexos o sobre colectivos minoritarios (Szirom, 2017; Santos, 2015).

Con este contexto previo, no consideramos efectivo establecer un debate inclusivo sobre el sexismo (Becker \& Barreto, 2014) que pueda ser productivo en términos de Educación para la Ciudadanía, entendiendo por productivo aquel que lleva la creación autónoma de una postura razonada y no a la imposición docente, peligro especialmente común en contextos universitarios (Biesta, 2017; Jefferess, 2016; Buller, 2015).

Probablemente, por el claro desequilibrio en la razón de sexos de la muestra, la mayoría de críticas al status quo sobre el feminismo y su lucha se asocian a conductas masculinas improcedentes, algo a todas luces evidente; sin embargo, hay una clara falta de autocrítica en cuanto al papel de la mujer en este contexto, entendido en un sentido emancipador, positivo y activo (Hirschmann, 2018; Hirsch y Keller, 2015). Así, por ejemplo, del listado de conceptos clave para abordar el feminismo ofrecido por Pilcher y Whelehan (op. cit.), una fracción inferior a la sexta parte ha sido citados sea a través del propio vocablo de un sinónimo claro.

Cabe señalar que una estrategia útil para la mejora de la eficiencia en los debates de esta naturaleza pasa por una formación conceptual e histórica más consolidada. Sin embargo, hoy día la primacía del diálogo y la comunicación suele hacerse al precio de una fase previa e individual de conocimiento y reflexión sobre la naturaleza de los hechos a debatir (Rose, 2013; Titus, 2000; Finke, 1993).

Respecto al desarrollo de la segunda fase, la charla sobre el sexismo diario expuesto a través de los casos recopilados, en el portal creado al efecto por Laura Bates, comprueba en buena medida la consecuencia de lo anterior: pese a que existe un alto grado de compromiso y apoyo respecto a la superación de agravios comparativos entre sexos, así como sobre las prácticas denigrantes o claramente inaceptables que aún hoy día las mujeres de todo el mundo documentan a través de la citada web, no se observa una reflexión articulada y profunda sobre los procesos que desencadenan dichos efectos.

Desde una perspectiva científico-educativa, la acumulación de calificativos de indignación y rechazo desarticulados de factores explicativos precisos (más allá de dicotomías del tipo bueno/malo), así como de un lenguaje más conciso es algo que necesita ser replanteado por dos motivos: 
- Porque solo un pensamiento científico posibilita un análisis objetivo de los antecedentes, los procesos y sus causas, constituyéndose como como un instrumento intelectual insustituible de entendimiento con el mundo.

- Y porque sólo procediendo de forma eficiente se pueden alcanzar soluciones eficientes, de modo que tratar como cajas negras (Heyes, 2016) los temas sociales a debatir, aunque sea una práctica de los tiempos, no debe pasar silenciada del mismo modo en los contextos formativos, muy especialmente en educación superior.

El sexismo como práctica es percibido pero no definido, lo que demuestra la existencia de concepciones muy subjetivas y de escaso contraste interpersonal. Desde quienes plantean esta realidad en tanto que práctica material a quienes la amplían hasta el ámbito de las elecciones personales, o quienes no aceptan la diferenciación mórfica como una disociación concomitante de virtudes y defectos; así, se constata la existencia de una idea vaga que sólo en sus expresiones más flagrantes se demuestra unívoca.

Esto explica la pobreza conceptual general, así como la orientación de los focos de interés hacia el escándalo de las situaciones más escabrosas que hacia el análisis explicativo sosegado que debe antecederlo: durante la charla, aunque de forma no especialmente enfática, la autora utiliza algunos conceptos propios del movimiento feminista pertinentes para el análisis del sexismo, pero que sin embargo no son recogidos de forma recurrente en los comentarios y réplicas de las personas participantes, como dicotomía, doble estándar, estereotipo o patriarcado.

\section{CONCLUSIONES}

El estudio ha permitido señalar la necesidad de conocer las bases epistemológicas del discurso feminista incluso en contextos, como el abordado, que caben suponer especialmente sensibilizados. Existe una conciencia activa, alerta, pero no organizada y definida respecto al sexismo: todas las participantes del estudio manifestaron haber sido agredidas sexualmente en algún momento de su vida. La combinación de ambas realidades explica, al menos en parte, la polémica y ambigüedad que con frecuencia pueblan los debates de esta índole.

Por ello, consideramos especialmente necesario seguir profundizando en estrategias didácticas que permitan la enseñanza de las corrientes de pensamiento y movilización social contemporáneas, de una forma detallada conceptualmente y abierta ideológicamente, en tanto que, como docentes, nunca debemos perder de vista que nuestro compromiso es el de hacer aprehensible el conocimiento a las nuevas generaciones, pero no imponer nuestro juicio al respecto. Algo muy común en la lógica de bandos que temas tan candentes como este suelen provocar y, de hecho, provocaron en el aula.

Encontrar la forma para que desde la construcción del criterio personal de quien aprende se produzcan espacios de encuentro y consenso no es fácil, pero es precisamente esto lo que lo hace necesario. La diversidad de opiniones sobre qué constituye una práctica sexista no es ni buena ni mala, sino una realidad. Ayudar a transformarla en un espacio de encuentro con elementos comunes, por mínimos que sean, no puede hacerse al precio de la imposición, por mucha convicción de certeza que se tenga. Intentarlo sólo va a derivar en estrategias estudiantiles históricas de supervivencia y a desplazar el foco de interés hacia cuestiones progresivamente menos relevantes en cuanto a criterios de causalidad y contexto.

El estudio también ha puesto de manifiesto algunos problemas de diseño o de transmisión de la intencionalidad, motivo por el que la clasificación de anotaciones mediante el sistema de etiquetas ha presentado algunas incidencias, siendo la más reseñable la no categorización de un número significativo de las mismas; así como cierta confusión en cuándo a cómo se debe proceder para clasificar la propia proposición como positiva o negativa. En parte esto encuentra su explicación en el aparto tecnológico utilizado, de carácter excepcional sobre todo por el uso de etiquetas; pero también debido a la dificultad de separar la componente emocional de la racional a la hora de emitir juicio sobre los ejemplos planteados por la conferenciante. 
Una asignatura como la utilizada, Educación para la Ciudadanía, tiene ante este tipo de retos la verdadera legitimación de su existencia, si se observan las cuestiones señaladas. La formación integral de la ciudadanía, formalizada curricularmente desde la educación superior, no puede ser ajena a este tipo de realidades. Por ello, es al desarrollo de propuestas en este sentido al que, entendemos, merece la pena seguir dedicándole conocimiento e interés.

\section{Referencias}

Alavi, S. M., Akbarian, I. (2012). The role of vocabulary size in predicting performance on TOEFL reading item types. System, 40(3), 376-385. DOI: 10.1016/j.system.2012.07.002

Ali, S. (2013). The myth of 'Everyday Sexism'. Spiked, 24 de octubre. Recuperado de https://goo.gl/MavyMe.

Becker, J. C. y Barreto, M. (2014). Ways to go: Men's and women's support for aggressive and nonaggressive confrontation of sexism as a function of gender identification. Journal of Social Issues, 70(4), 668-686.

Biesta, G. (2017). The rediscovery of teaching. Londres: Routledge.

Brown, B. (2015). Destructive feminism of Everyday Sexism Project tears men and women apart. The conservative woman, 18 de junio. Recuperado de https://goo.gl/QD4xSc.

Buller, J. L. (2015). Change leadership in higher education: A practical guide to academic transformation. John Wiley \& Sons.

Cámara Marín, S. (2014). Análisis de la cultura de género en el ámbito educativo. Lisboa: FCSH: DS - Dissertações de Mestrado. (Dissertação apresentada para cumprimento dos requisitos necessários à obtenção do grau de Mestre em Estudos sobre as Mulheres). URI: http://hdl.handle.net/10362/13919

Cepeda, H., Correa, K., Lozano, E. y Urquizo, D. (2018). Análisis crítico del conductismo y constructivismo, como teorías de aprendizaje en educación. Revista Órbita Pedagógica, 4(1), 01-12.

Cornejo, M. y Salas, N. (2011). Rigor y calidad metodológicos: un reto a la investigación social cualitativa. Psicoperspectivas, 10(2), 12-34. DOI: 10.5027/psicoperspectivas-Vol10-Issue2fulltext-144.

Diller, A. (2018). The gender question in education: Theory, pedagogy, and politics. Routledge.

Fernández Enguita, M. (2016). La educación en la encrucijada. Madrid: Fundación Santillana.

Finkel, L. (1993). Knowledge as bait: Feminism, voice, and the pedagogical unconscious. College English, 55(1), 7-27.

Gyllstad, H., Vilkaite, L. y Schmitt, N. (2015). Assessing vocabulary size through multiple-choice formats: Issues with guessing and sampling rates. ITL-International Journal of Applied Linguistics, 166(2), 278-306.

Gray, E., Blaise, M. y Knight, L. (2017). A different kind of academic performance: Using the arts to address sexism in Australian universities. EduResearch Matters, 8.

Heyes, C. (2016). Blackboxing: social learning strategies and cultural evolution. Phil. Trans. R. Soc. B, 371: 20150369. DOI : 10.1098/rstb.2015.0369

Holt, J. (1983). How Children Learn. Nueva York: Delacorte Press.

Holt, J. (1982). How Children Fail. Nueva York: Merloyd Lawrence Books.

Hammond, M., Milojev, P., Huang, Y. y Sibley, C. G. (2017). Benevolent sexism and hostile sexism across the ages. Social Psychological and Personality Science, 1-12. DOI: $10.1177 / 1948550617727588$.

Hirsch, M. y Keller, E. F. (2015). Conflicts in feminism. Routledge.

Hirschmann, N. J. (2018). Rethinking obligation for feminism. En Revisioning the political (pp. 157-180). Routledge. 
Jefferess, D. (2016). Relation and action in global citizenship education. Globalization and Global Citizenship: Interdisciplinary Approaches, 11.

Jiménez Rodrigo, M., Román Onsalo, M., y Traverso Cortés, J. (2011). Lenguaje no sexista y barreras a su utilización. Un estudio en el ámbito universitario. Revista de investigación en educación, 2(9), 174-183.

Jones, S. y Hughes, H. (2016). Changing the place of teacher education: Feminism, fear, and pedagogical paradoxes. Harvard Educational Review, 86(2), 161-182.

Lerena, C. (1983). Reprimir y liberar. Crítica sociológica de la educación y de la cultura contemporáneas. Madrid: Akal.

Maeso Fernández, M. E., Salamanca Castro, A. B., Sánchez Castro, S., Gil Pascual, J. A., Amézcua Sánchez, A. y Ayuso Medina, N. (2015). Ambivalent sexism level in students of first course of Secondary Compulsory Education in Madrid city. Journal of feminist, Gender and Women Studies, 2, 23-31.

Martínez-Alemán, A. M., Pusser, B. y Bensimon, E. M. (Eds.) (2015). Critical approaches to the study of higher education: A practical introduction. JHU Press.

Martínez Romera, D. (2018). Evaluar el pensamiento crítico en Educación para la Ciudadanía: propuesta para contextos masificados. Didacticae, 3, 131-144.

Mingo, A. y Moreno, H. (2015). El ocioso intento de tapar el sol con un dedo: violencia de género en la universidad. Perfiles educativos, 37(148), 138-155.

Nogueira, L., Nogueira, M., Navarro, J. y Lizcano, E. (2015). Metodología de las ciencias sociales. Barcelona: Tecnos.

Padilla Carmona, M., Sánchez García, M., Martín Berrido, M. y Moreno Sánchez, E. (1999). Análisis de estereotipos sexistas en una muestra de estudiantes de CC. de la Educación. Revista de Investigación Educativa, 17(1), 127-147.

Pilcher, J. y Whelehan, I. (2016). Key concepts in gender studies. UK: Sage.

Quinn, J., Davies, S. y Lubelska, C. (2017). Changing the subject: Women in higher education. Taylor \& Francis.

Quivy, R. y Campenhoudt, L. (2006). Manual de investigación en Ciencias Sociales. México, D. F.: Limusa.

Ronai, C. R., Zsembik, B. A. y Feagin, J. R. (2014). Everyday sexism in the third millennium. Nueva York: Routledge.

Rose, G. (2013). Feminism and Geography: The Limits of Geographical Knowledge. Nueva York: John Wiley \& Sons.

Santos, S. A. (2015). School-based sex education under the spotlight of sexual and intimate citizenship: A focus on Portugal and England (Tesis doctoral). Universidade do Porto, Oporto (Portugal).

Schmitt, N. (2014). Size and depth of vocabulary knowledge: What the research shows. Language Learning, 64(4), 913-951. DOI: 10.1111/lang.12077

Szirom, T. (2017). Teaching Gender? Sex Education and Sexual Stereotypes (Vol. 21). Taylor \& Francis.

Titus, J. (2000). Engaging student resistance to feminism: 'How is this stuff going to make us better teachers?' Gender and Education, 12(1), 21-37.

Woolfolk, A., Winne, P., Perry, N. y Shapka, J. (2010). Educational Psychology. Toronto: Pearson.

\section{CÓMO CITAR ESTE ARTÍCULO}

Martínez Romera, D. D. (2019). Feminismo y percepción del sexismo mediante TIC en educación superior. Un estudio de caso. Didáctica de las ciencias experimentales y sociales, 36, 3-16. DOI: 10.7203/DCES.36.12634. 